\title{
Handover Analysis for Multicast Enabled Network Mobility Management Using Network Simulator
}

\author{
Azana Hafizah Mohd Aman ${ }^{1}$, Aisha-Hassan A. Hashim ${ }^{2}$, Huda Adibah Mohd \\ Ramli and Shayla Islam \\ Kulliyyah of Engineering, International Islamic University Malaysia, Jalan \\ Gombak 53100, Kuala Lumpur, Malaysia \\ 'azana05@yahoo.com,2aisha@iium.edu.my
}

\begin{abstract}
Network mobility management has become a popular topic in networking research due to its ability to mitigate mobile IPv6 problems. However the standard network mobility management does not support multicast traffic. Hence in this paper, we introduce context transfer and multicast fast reroute, to enable multicast in the standard network mobility management. This implementation enables multicast in network mobility management with high handover performance support. This paper evaluates handover performance for multicast enabled network mobility management using network simulator called NS3. NS3 is a network simulator that implements virtual network prototype. By using the virtual network entities provided by the NS3, the proposed network mobility management architecture is simulated and its activity is analyzed. The parameters used are referred to real network implementation.
\end{abstract}

Keywords: Multicast, NS3, Network Mobility, Handover

\section{Introduction}

Multicast mobile IP has been an interest in recent years but there is as yet no standard solution. According to [1-3], by year 2019, IP traffic will surpass two zettabyte threshold, with mobile traffic grows three times quicker than static IP traffic. Network mobility is known as changing network layer while moving in a network. The initial standard method of network mobility is described in Mobile IPv6 (MIPv6) [6]. This standard method suffers from slow handover due to multicast routing and inefficient overhead [4-5]. It also suffers from delay due to triangular forwarding [4-5]. As a resolution to this problem, this paper offered improvements in terms of high data delivery in network mobility management. The improved method is an integration of two methods. These methods are implemented in network mobility management called Proxy Mobile IPv6 (PMIPv6) [7-8]. The two methods are Context Transfer [9-11] and Multicast Fast Reroute [12]. The novel improved method is named as CTMFR. This implementation helps to shortened the distribution time of the essential multicast data hence improves the handover performance. The implementation and evaluation of the new improved method is through Network Simulator known as NS3. The new improved method is compared to the standard method of PMIPv6.

The organization of this paper is as follows. Section 2 describes recent network mobility management development. Followed by the proposed improvement method in Section 3. Then the simulation results and analysis in outlined in Section 4. As a final point, Section 5 concluded the paper. 


\section{Recent Network Mobility Management Development}

Recent proposals to enable multicast in PMIPv6 can be grouped as route optimization, global mobility, load balancing and context transfer. These proposals are evaluated using either mathematical analysis or simulator analysis. Generally a network simulator is an application that predicts the events of a network [14-15]. Network devices are depicted with nodes, links, devices and etc. While simulation is a testing of the depicted network entities on a network simulator [14-15]. Simulation of network is difficult, as it is not simple to develop network event, such as handover, multicast or mobility. A network emulator allows users to host real devices and applications into a virtual test network [1415]. A network simulator must be able to model and configure network entities, topologies and able to provide network results in values and graphs. Figure 1 shows the basic network model for PMIPv6.

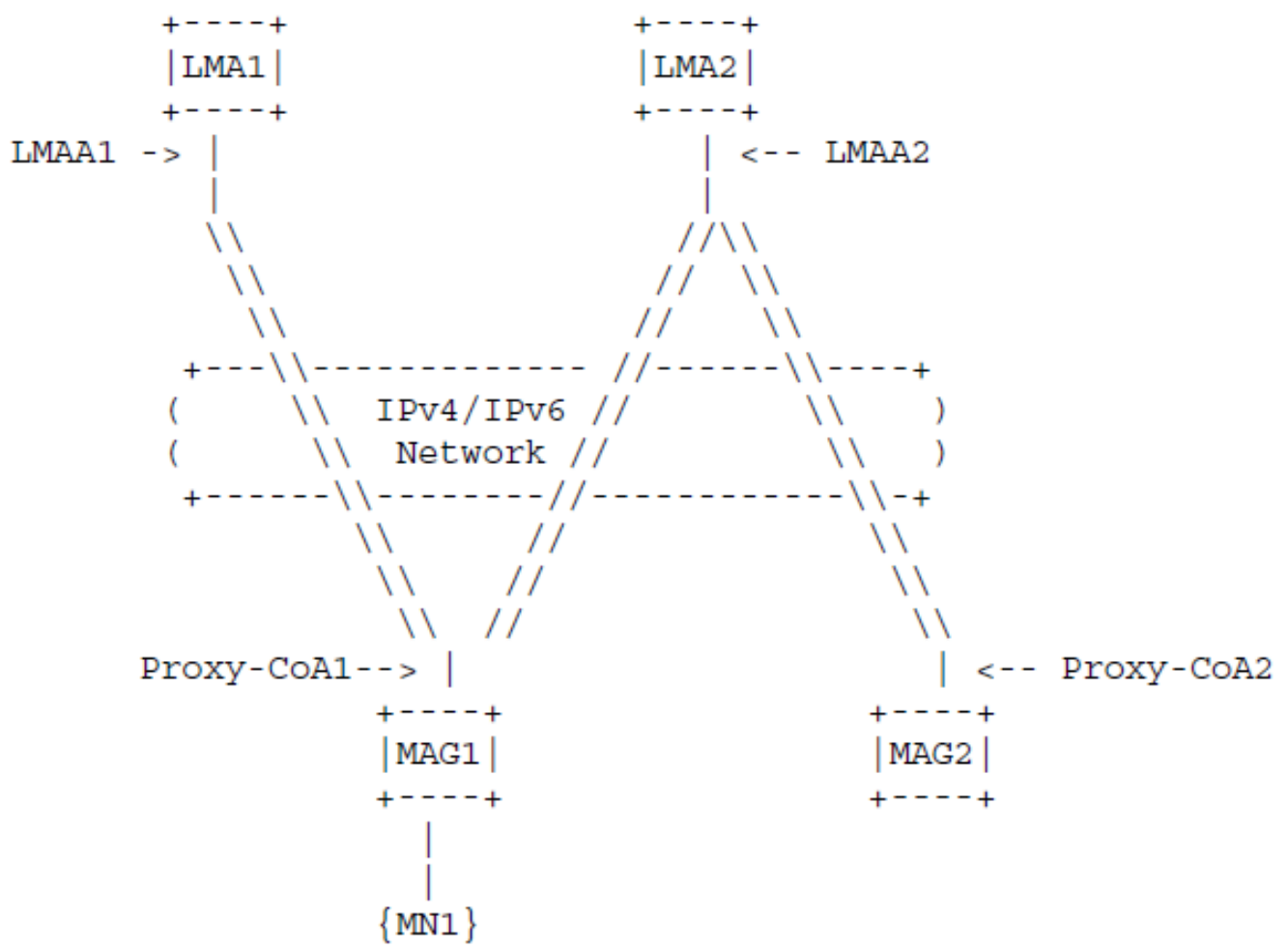

Figure 1. Network Model of PMIP v6 [7-8]

In [16], the performance of PMIPv6 protocol is studied using Network Simulator (NS) version 2.29. It extended the existing MIPv6 to create PMIPv6 environment. Total number of 7 nodes including 1 mobile node are configured. Transmission Control Protocol (TCP) and User Datagram Protocol (UDP) are the traffic configured. Performance metrics measured are throughput, average handover delay and average packet delivery rate. Data collected are number of packets and number of successfully distributed bytes and also number of packets sent. The suggested design satisfied the flow necessities for PMIPv6, however there is no multicast traffic included.

Multi-Protocol Label Switching (MPLS) using OMNET++ network simulation for PMIPv6 is evaluated by [17]. Some modifications of the existing codes are done. xMIPv6 is the existing code, a base to support MPLS/PMIPv6 protocol. Performance metrics considered are end-to-end delay, handover overhead and delay. Variety number of hop between MAG and LMA were set for the simulation. Scenario manager, configurator and channel control modules of OMNET++ are used to support the network model. It verified that the proposed architecture satisfied the flow mobility standards of PMIPv6. Work in 
[19] improved flow mobility support using the NS3 network simulator. By using these NS3 functions, it applied the multi-interfaced for the host and the mobility process in the recommended design. It proved that the recommended architecture fulfilled the mobility standards.

As for [18] and [20] both covers the security part of network mobility management. Hence, [18] implemented authorization, authentication and accounting (AAA) server in PMIPv6 topology. The AAA server and PMIPv6 implementation is via NS-2.29. Performance metric considered are handover delay and handover. Results are calculated based on varying number of LMAs. It certified that the offered scheme fulfilled the mobility desires of PMIPv6. While work in [20], suggested a secure handover mechanism for PMIPv6 environment. It executed group key ticket for fast re-authentication using NS2. Performance metrics measured are packet loss, handover latency and signaling cost. It proved that the proposed architecture pleased the flow mobility standards of PMIPv6.

By using NS3, work in [21] described PMIPv6 entities. It applied MIPv6 module in NS3 as the basic module. Performance metric measured are packet drop and handover latency. The values are collected in a module called PCAP. Another [22] considered the performance of PMIPv6 protocol using QUALNET. In order to apply PMIPv6 module, it implemented traffic control by ranging the queuing function of the QUALNET. Performance metrics considered are packet buffering and packet loss. Even though work in [21] and [22] satisfied the flow mobility requirements of PMIPv6, there is no multicast traffic included.

\section{Proposed Multicast Network Mobility Management}

Improving PMIPv6 with multicast ability is an important topic to study. This is because, PMIPv6 basically allows unicast mobility and no consideration towards multicast mobility. Hence, it is the aim of this work to introduce context transfer and multicast fast reroute to enable multicast in network mobility management. Both methods are configured and modified to suit the standard network mobility management. This implementation enables multicast to network mobility management with high network performance. Besides enabling multicast, this method address the handover performance issues related to PMIPv6 multicast environment.

Using this method, the context is successfully transferred in advanced. This reduces unnecessary transmission after the handover. The destination node which is known as Mobile Node (MN) is able to receive multicast data immediately after the handover. Figure 2 shows the basic network model for the proposed method while Figure 3 shows the signaling call flow for the proposed method. 


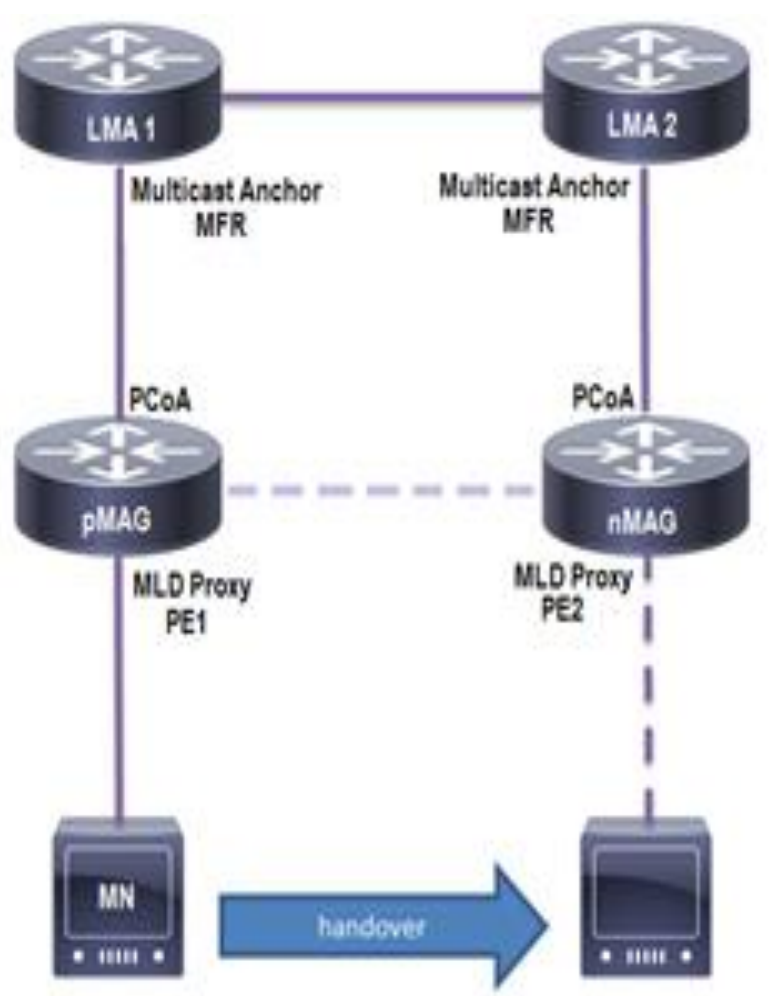

Figure 2. Basic Network Model for the Improved Method

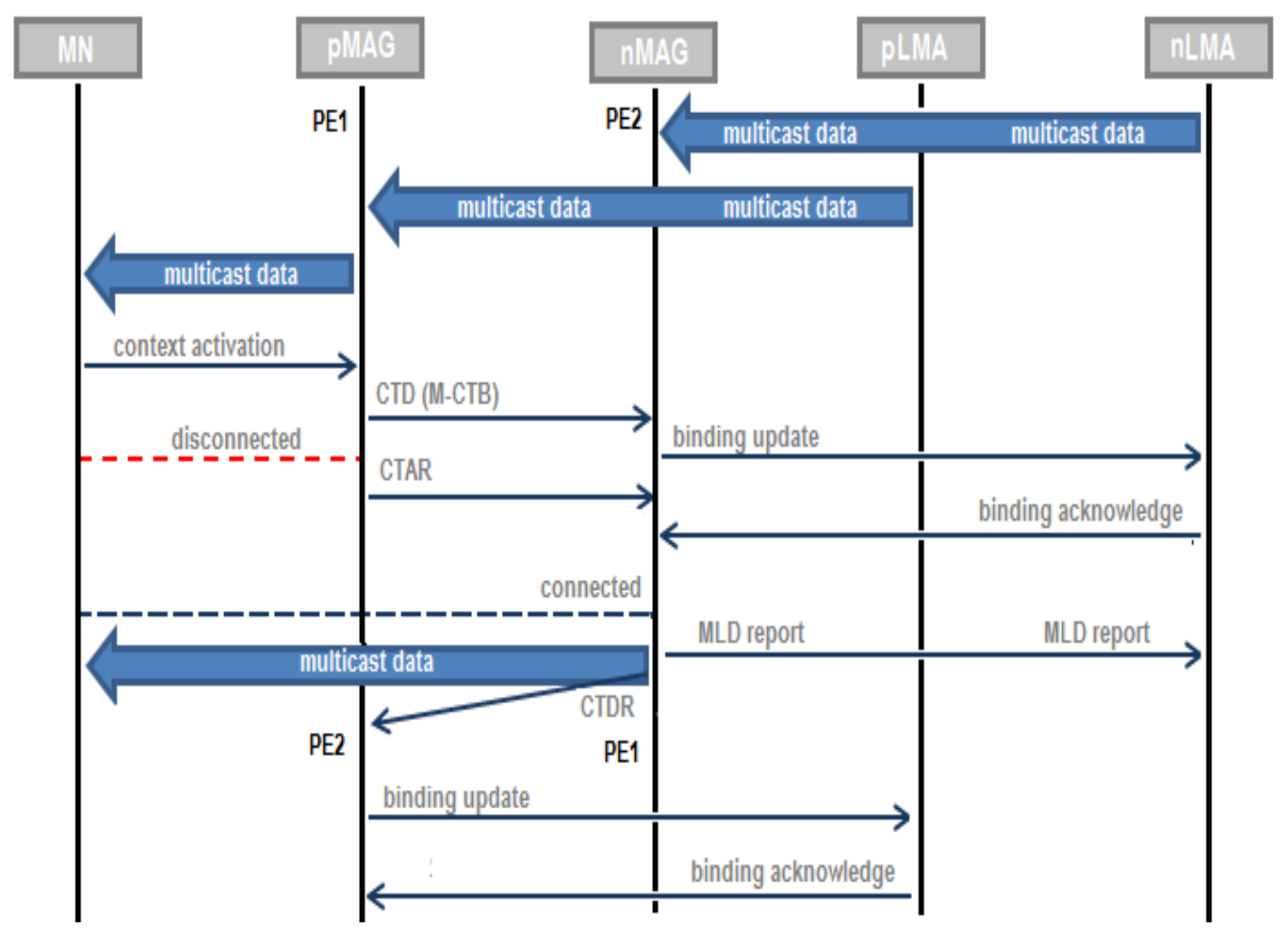

Figure 3. Process Flow for the Improved Method

From the above mentioned operation, it can be seen that by applying the methods, the steps needed to gain the service back to normal are minimized. Since the multicast context data is transported between the two MAGs before the handover is finished. Hence, all the data necessary for the multicast group is already transported. It is noted that the MN joins 
the multicast group immediately as the MN changes to the nMAG. The signaling cost is minimized due to localized communication of the MAGs. The MN is not involved in sending the group membership information to the nMAG or LMA. The nMAG receives the information needed in the multicast context transfer block that is sent by pMAG.

Figure 4 depicts the network detail for the NS3 simulation, while Figure 5 shows the NS3 network topology setup according to Figure 4 with total number of 18 nodes including $1 \mathrm{MN}$. Figure 6 shows part of the simulation of the topology.

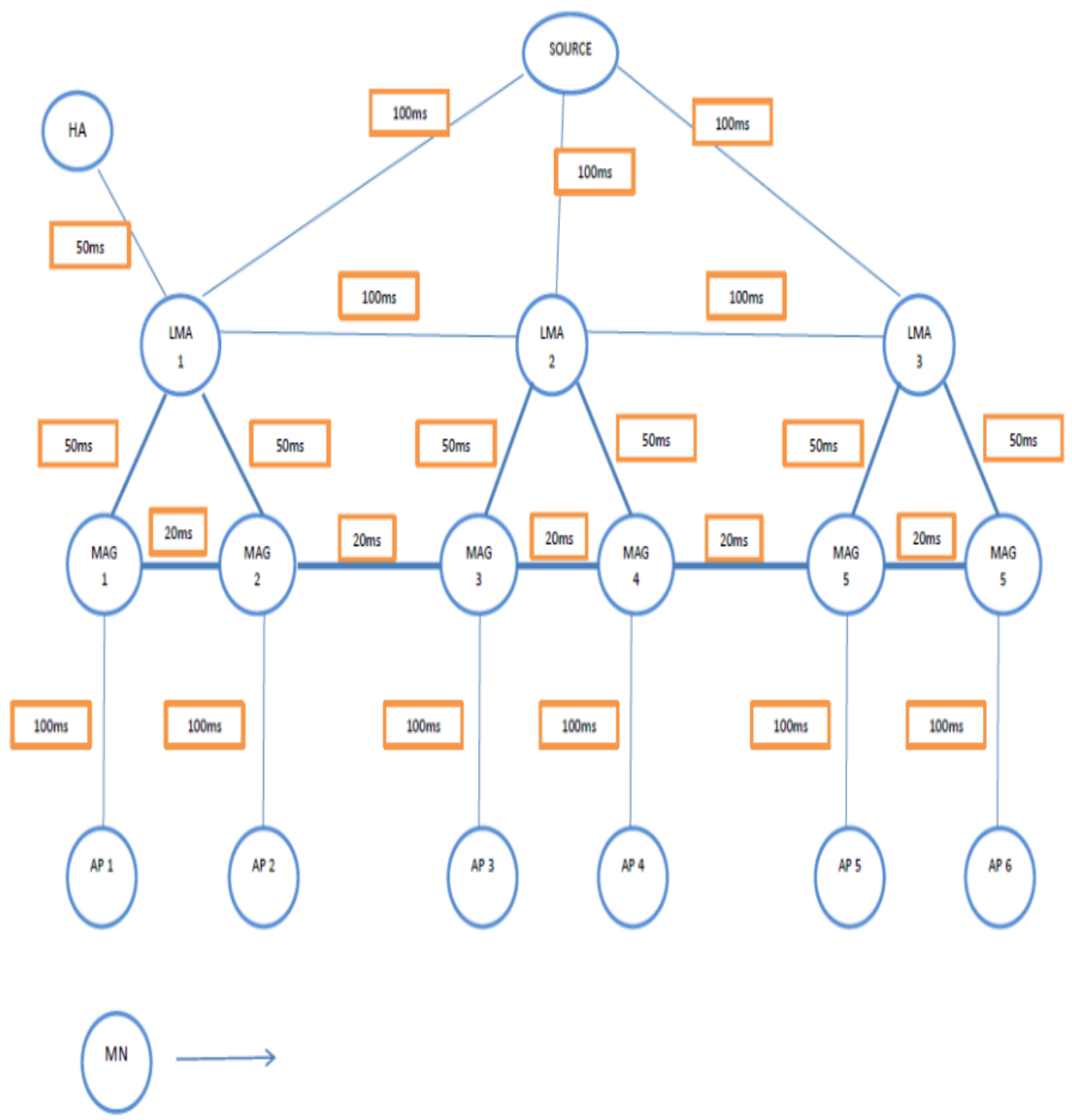

Figure 4. Network Topology for the Simulation 


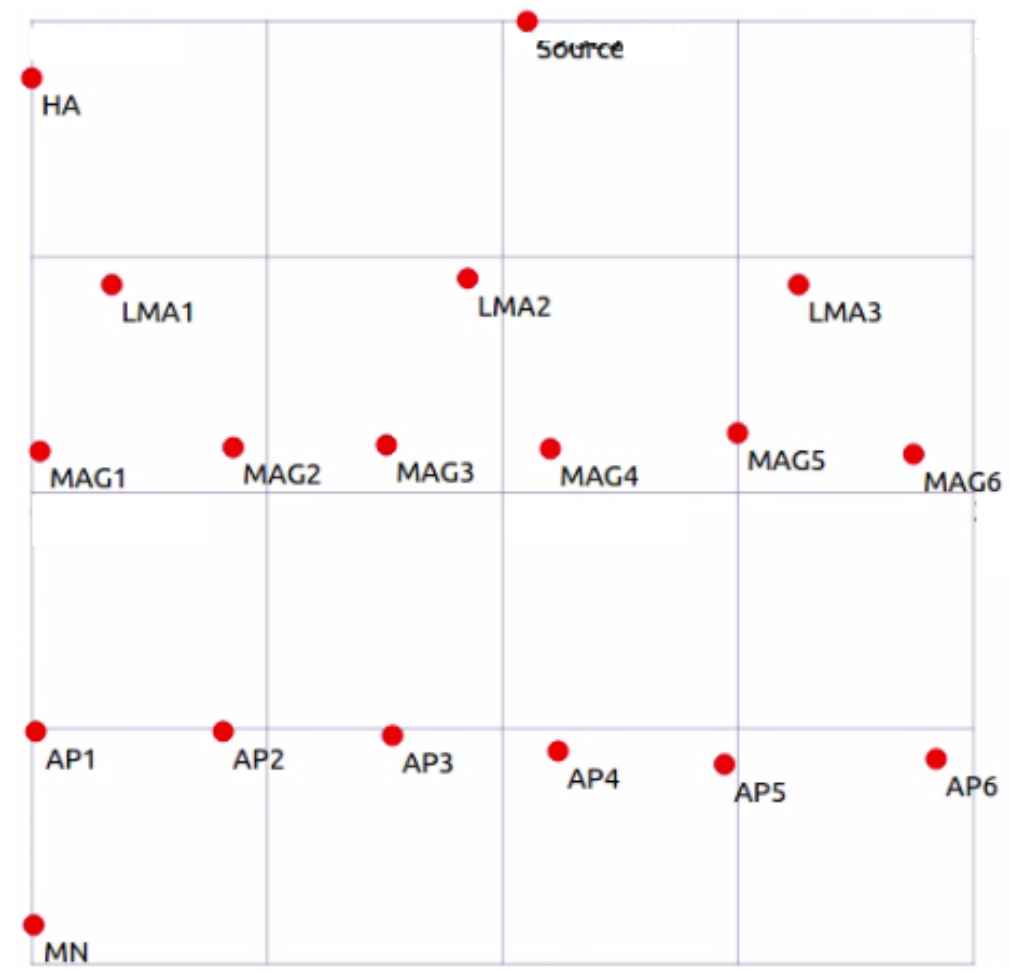

Figure 5. Simulation Topology Setup

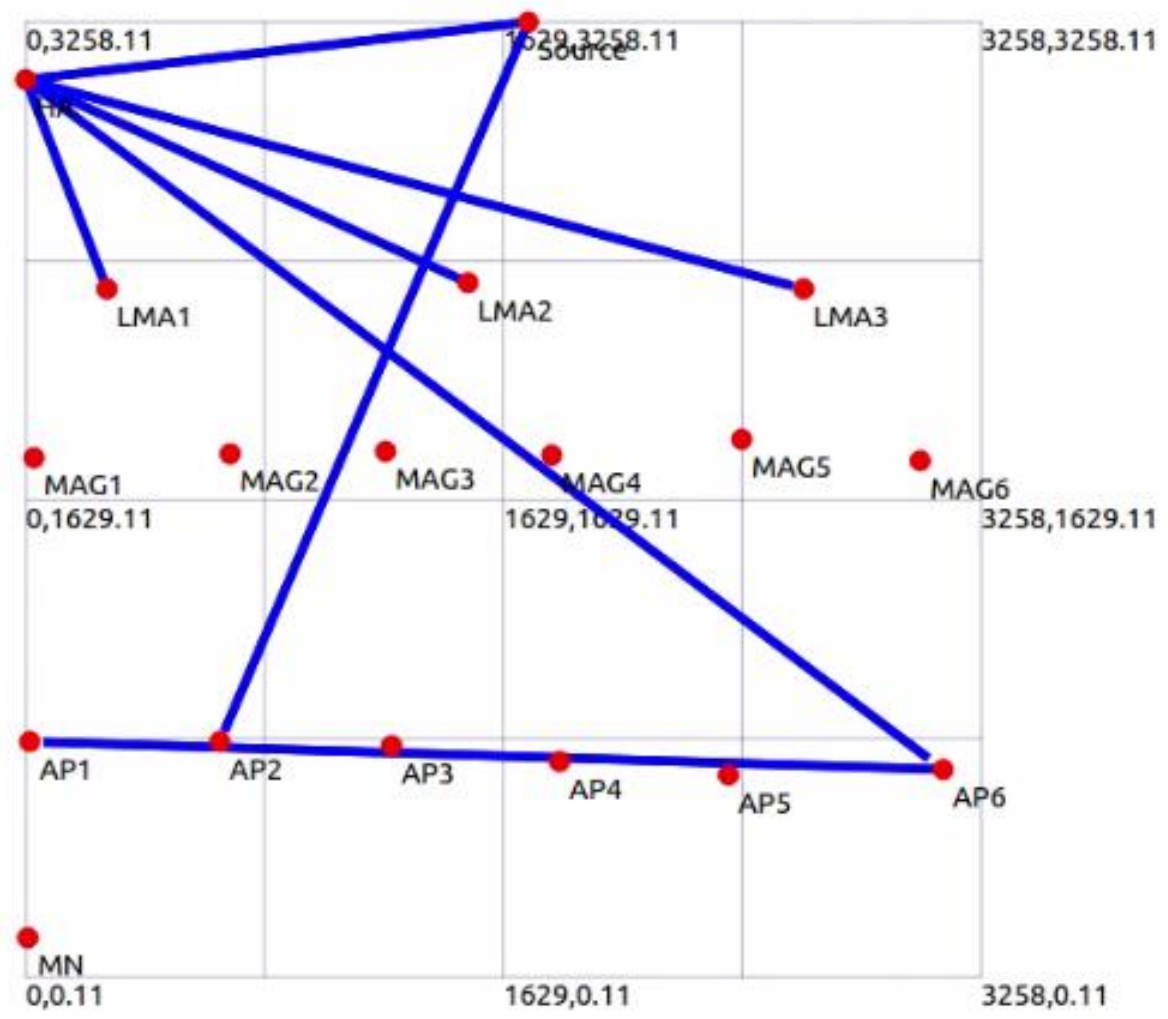

Figure 6. Simulation 


\section{Simulation Results and Analysis}

Node mobility certainly has a significant impact upon network performance. When a MN with an active flow moves from a network to other network, the path for data-flow also changes. Handover is defined as changing network connection of a MN. Handover latency is calculated by the time interval of the last packet established at the pMAG and the first packet established at the nMAG. Handover value is extracted from the trace-file produced during the simulation process using a trace-file analyzer.

To achieve seamless mobility, the handover latency value should be kept as low as possible. High handover latency may affect real-time communicating applications like voice/video calls and online gaming. The amount of satisfactory delay depends on the application since their acceptable rate differs from each other. For some applications, handover latency above a certain limits may cause service disconnection, leads to poor handover performance. The relationship between handover latency and network performance is inversely proportional to each other. Thus requires more sophisticated and promising means to overcome the problem. Hence minimizing handover latency is very crucial. Minimizing handover delay is valuable to throughput sensitive applications.

Figure 7 shows the handover latency versus time for the improvise method. While Figure 8 shows the comparison of the improvised method and for the standard method in terms of handover latency versus simulation time. This proved that by using PMIPv6 with CTMFR, the handover can be largely reduced.

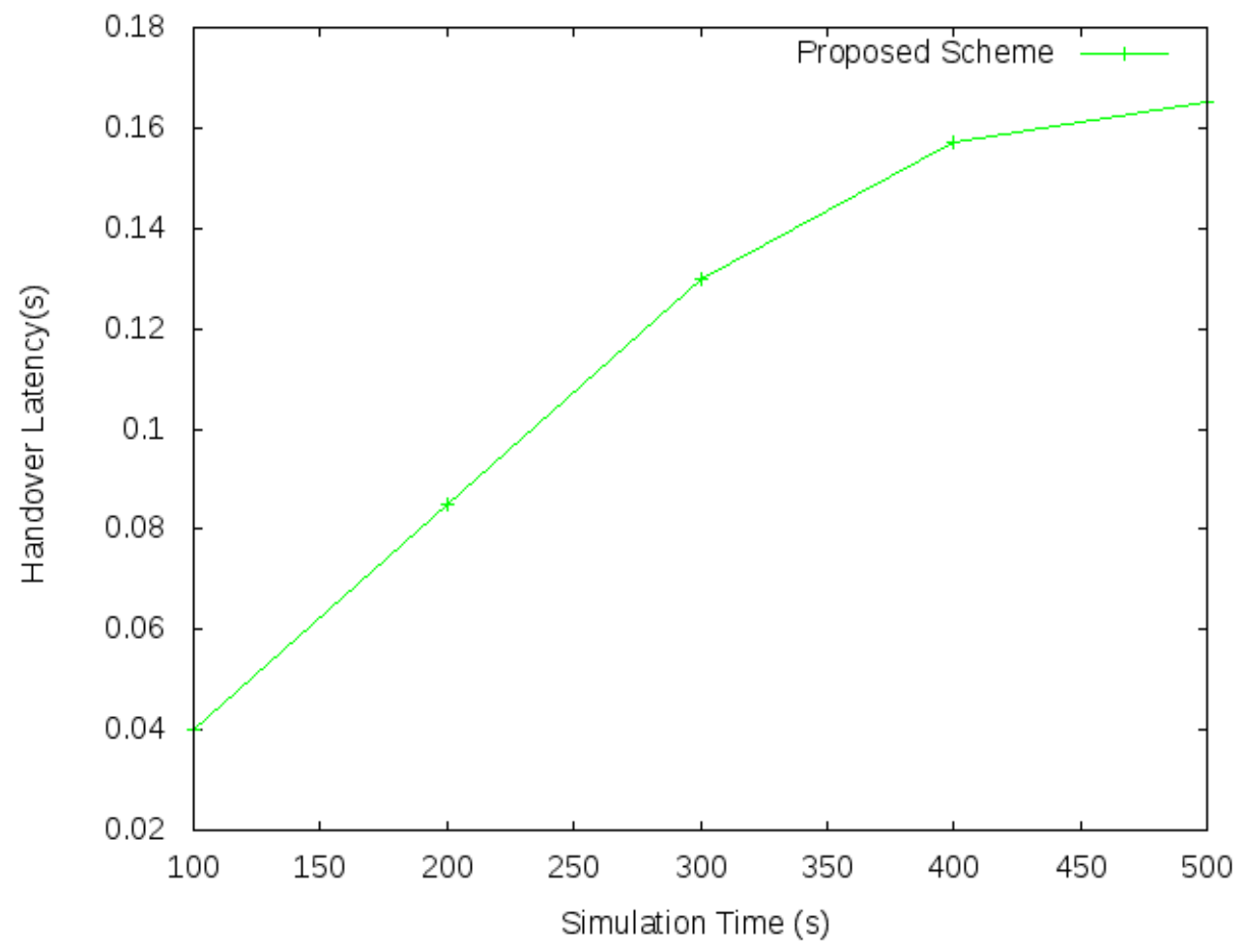

Figure 7. Handover Latency versus Simulation Time - Proposed Scheme 


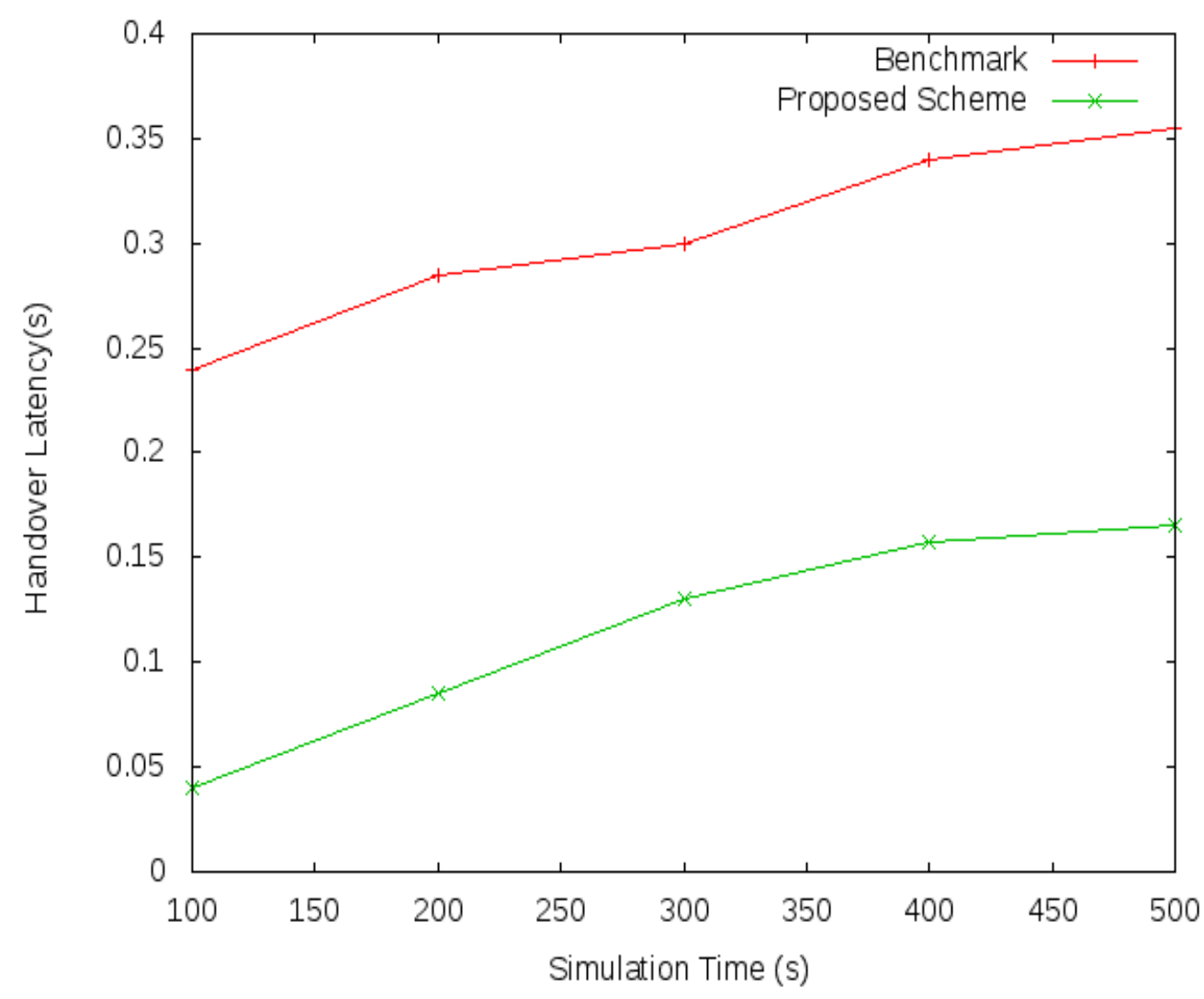

Figure 8. Handover Latency versus Simulation Time - Benchmark

The effectiveness of the applied method has been shown in the Figure 7 and Figure 8 . It can be seen that there is huge gap between the improvised method and the standard method as shown in Figure 8. By using PMIPv6 with CTMFR, the handover performance is $50 \%$ better than the standard method. When handover is reduced, MN does not have to wait for the long handover process to resume its multicast communication.

The context transfer multicast fast reroute simplifies the flow. Therefore leads to better handover performance. This enhancement permits two-ways context transfer design in multicast PMIPv6. Multicast communication, mainly the real-time video applications are heavily viewed channels. Since both MAGs are in the same multicast group, smooth handover with no connection break are implemented, thus traffic continuously running without any interruption.

\section{Conclusion}

The improvised method is evaluated using simulation approach. After describing the simulation setup, the performance results of the selected metrics are presented and validated. Compared to PMIPv6 standard method, the improvised method offers highly considerable better handover performance. From the simulation results, this proved that by applying the predictive CT and MFR, the enhanced method performed better than PMIPv6 and provides little performance issues. 


\section{References}

[1] C. D. Cisco Visual Networking Index: Global Mobile Data Traffic Forecast Update 2014 - 2019, Cisco White Paper, (2015).

[2] Cisco Visual Networking Index: Forecast and Methodology 2014 - 2019, Cisco White Paper, (2015).

[3] Cisco Visual Networking Index: The Zettabyte Era Trends and Analysis, Cisco White Paper, (2015).

[4] TC Schmidt, M Waehlisch and G Fairhurst, "Multicast mobility in MIPv6: problem statement \& brief survey," RFC 5757, (2010) February.

[5] CJ Bernardos, M Calderon and I Soto, "PMIPv6 and network mobility problem statement," InternetDraft, (2012) March.

[6] Johnson, D., Perkins, C., and J. Arkko, "Mobility support in IPv6," RFC 3775, (2004) June.

[7] Gundavelli S, Ed Leung, K Devarapalli, V Chowdhury K and B Patil, "Proxy Mobile IPv6(PMIPv6)," RFC 5213, (2008) August.

[8] Daehyeok Kim, Wan-Seon Lim and Young-Joo Suh, "Multicast extension to Proxy Mobile IPv6 for mobile multicast services," Journal of Computing Science and Engineering, vol. 5, (2011) December, pp. 316-323.

[9] D. von Hugo and H. Asaeda, "Context Transfer Protocol extension for multicast," Internet-Draft, (2013) February.

[10] J Loughney, M Nakhjiri, C Perkins and R Koodli, “Context Transfer Protocol,” RFC 4067, (2005) July.

[11] Tien-Thinh Nguyen, and Christian Bonnet, "Performance optimization of multicast content delivery in a mobile environment based on PMIPv6," Wireless Communications and Networking Conference (WCNC) IEEE, (2013) April, pp. 1249-1254.

[12] A. Karan, C. Filsfils, IJ. Wijnands, and B. Decraene, "Multicast Only Fasr Reroute (MoFRR)," RFC 7431, (2015) August.

[13] T. Schmidt, M. Waehlisch and S. Krishnan, "Base deployment for multicast listener support in Proxy Mobile IPv6 (PMIPv6) domains," RFC 6224, (2011) April.

[14] Saba Siraj, Ajay Kumar Gupta, Rinku-Badgujar, Network Simulation Tools Survey, International J. of Advanced Research in Computer and Communication Engineering Vol. 1(4), (2012).

[15] Jianli Pan. A Survey of Network Simulation Tools: Current Status and Future Developments.

[16] Farouk Abdul Jalin and Raed Alsaqour, A Simulation Study of Proxy Mobile IPV6 (PMIPV6) Protocol, J. of Engineering and Applied Sciences, vol. 11(7), (2016).

[17] Hatsadin Payappanon, Thossaporn Kamolphiwong, Kevin Robert Elz, Simulation and Evaluation of MPLS based PMIPv6 Network, International J. of Advances in Computer Science and Technology, Vol.2 (8), (2013) ,Pp: 07-11.

[18] Ashwini Prakash Ghatol*, Prof. N. M. Tarbani, Optimized Handover Mechanism for Proxy Mobile IPv6, International Journal of Advanced Research in Computer Science and Software Engineering IJARCSE, Vol. 5 (6), (2015).

[19] Hyon-Young Choi, Sung-Gi Min, Youn-Hee Han and Rajeev Koodli, Design and Simulation of a Flow Mobility Scheme Based on Proxy Mobile IPv6, J Inf Process Syst, Vol.8 (4), (2012).

[20] K.Mayuri and K.S.Ranjith, A Novel Secure Handover Mechanism In Pmipv6 Networks, International J. of Information Technology Convergence and Services, Vol.4 (4), (2014).

[21] Hyon-Young Choi, Sung-Gi Min, Youn-Hee Han, Jungsoo Park and Hyoungjun Kim,Implementation and Evaluation of Proxy Mobile IPv6 in NS-3 Network Simulator, IEEE Xplore (2011).

[22] Takeshi Usui, Designing Improved Traffic Control in Network-based Seamless Mobility Management for Wireless LAN, The Third International Conference on Advances in Future Internet (2011).

[23] JC. Zuniga, LM.Contreras, CJ. Bernardos, S. Jeon, and Y. Kim, "Multicast Mobility Routing Optimizations for Proxy Mobile IPv6", RFC 7028, (2013) September.

[24] L. Wang, S. Goa, H. Zhang, TC. Schmidt nad J. Guang, "Mobile multicast source support in PMIPv6 networks" Journal on Wireless Communications and Networking. (2013) June.

[25] Prithiviraj, A.; Krishnamoorthy, K.; K. Jaya Bharathy "Optimizing handover performance for heterogeneous wireless networks in pmipv6" International Journal of Advanced Research in Computer Science . (2014) Jan/Feb, Vol. 5 Issue 1, p89-92. 4p.

[26] S. Verma, GS Tomar, "Call Admission Control and Handoff Techniques for 3-G and Beyond Mobile Network", Asia-pacific Journal of Multimedia Services Convergence with Art, Humanities and Sociology, Vol.1, No.1, pp. 31-42 (2011).

[27] BJ. Majerick, GJ. Heijenk, A. Pras, and PT. de Boer, "Lossless Multicast Handovers in Proxy Fast Mobile IPv6 Networks" University of Twente, (2015) February.

[28] S., Kim, Y., "Deployment Models for Distributed Mobility Management", draft-sijeon-dmmdeployment-models-02 (work in progress), (2016) March.

[29] Moneeb Gohar, Sang Il Choi, Seok Joo Koh, "Fast handover using multicast handover agents in PMIPv6-based wireless network," The 25th International Conference on Information Networking (ICOIN 2011), (2011) January.

[30] Adnan J.Jabir, S. Shamala and Z.Zuriati, "A new strategy for signalling overhead reduction in the proxy mobile IPv6 Protocol,” Am. J. of Applied Sci., vol. 9, issue 4, (2012) , pp. 535-541. 
International Journal of Multimedia and Ubiquitous Engineering Vol.12, No.3 (2017) 